\title{
ESTUDO DA INFLUENZA A(H1N1) APLICADA AO MODELO SIRS
}

\author{
Caio L. T. F. Jardim Letícia A. F. Ferreira* ${ }^{*} \underline{\text { Dérek B. Prates }}^{\dagger}$ \\ Instituto de Ciência, Engenharia e Tecnologia, ICET, UFVJM, \\ 39803-371,Teófilo Otoni, MG \\ E-mail: caiodt4@hotmail.com, letty_ali@hotmail.com, derekbomfim@hotmail.com
}

\author{
Jaqueline M. Silva \\ Universidade Federal dos Vales do Jequitinhonha e Mucuri - Instituto de Ciência e Tecnologia \\ 39803-371, Campus Mucuri, Teófilo Otoni, MG \\ E-mail: jaqueline.silva@ufvjm.edu.br
}

$\underline{\text { RESUMO }}$

\section{Introdução}

Entre as diversas aplicações da modelagem matemática enquadram-se os estudos de modelos epidemiológicos que são importantes de serem estudados em função do grande impacto que tiveram na evolução da ciência, apresentando altas taxas de mortalidade humana. Por exemplo, na Peste Negra, uma pandemia de peste bubônica que assolou a Europa no século XIV, estima-se que morreram aproximadamente 70 milhões de pessoas. Estudos epidemiológicos se mostram necessários e úteis para a elaboração de medidas de precaução e políticas de saneamento. A modelagem epidemiológica se destaca como uma poderosa ferramenta de estudo e análise de tais epidemias e apresenta uma ampla variedade de técnicas que podem ser aplicadas tanto a casos gerais quanto a casos específicos.

Dentre os modelos mais usados em epidemiologia, destaca-se o pioneiro SIR, que foi proposto por Kermack e McKendrick em 1927. A construção do modelo baseou-se na divisão de uma população, considerada constante, em três classes: suscetíveis, infectados e removidos. A partir dessa linha, Kermack e McKendrick formularam o sistema de equações característico desse modelo e criaram o Teorema do Limiar, um marco na análise das epidemias que até hoje serve como forte aparato para a dedução de novos modelos.

Por outro lado, o modelo SIRS é uma variação do modelo SIR que considera a situação em que a população dos removidos perde sua imunidade e volta a ser considerado suscetível, após certo tempo(Oliveira, 2008). Apresenta um caráter mais dinâmico e representa de maneira mais fiel o comportamento de uma epidemia. Neste trabalho, o modelo SIRS será aplicado à uma epidemia da Influenza A(H1N1), doença que chamou a atenção mundial por ser uma das primeiras pandemias do século XXI, matando milhares de pessoas pelo mundo e infectando um número ainda maior.

Palavras-chave: Epidemiologia, SIRS, Influenza

\section{Métodos}

*bolsista de Iniciação Científica FAPEMIG/UFVJM

†bolsista de Iniciação Científica PIBIC/LNCC 
O modelo SIRS é um pouco mais representativo que o modelo SIR por apresentar situações que se aproximam melhor da realidade, com variação da população ao longo do tempo e a possibilidade de novo contágio dos indivíduos pela doença. O modelo admite a existência de três taxas: a taxa de variação temporal dos indivíduos suscetíveis, a taxa de variação temporal dos indivíduos infectados e a taxa de variação temporal dos indivíduos removidos. Os parâmetros da epidemia de Influenza $\mathrm{A}(\mathrm{H} 1 \mathrm{~N} 1)$ considerados no modelo são: $\alpha$ a taxa de mortalidade pela doença, $\beta$ a taxa de natalidade, $\mu$ a taxa de mortalidade natural, $\lambda$ é a taxa de infecção, $\gamma$ a taxa de recuperação e $\delta$ é a taxa a que os indivíduos recuperados voltam a se tornar suscetíveis. Este trabalho propõe duas hipóteses:

८) Não há mortes de indivíduos pela doença, ou seja, $\alpha=0$.

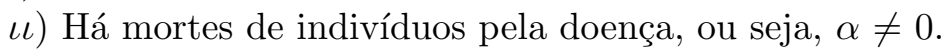

Pressupõe-se no modelo que a população varia ao longo do tempo e analisa-se a releitura do modelo SIRS em função das suas taxas de natalidade e mortalidade. Através da normatização dos dados e sendo $s, i$ e $r$, respectivamente, as frações da população de indivíduos suscetíveis, infectados e removidos, o modelo pode ser descrito da seguinte forma:

$$
\left\{\begin{array}{l}
\frac{d s}{d t}=(\alpha-\lambda) s i-(\beta+\delta) s-\delta i+\beta+\delta \\
\frac{d i}{d t}=i(\lambda s-(\alpha+\beta+\gamma)+\alpha i) \\
\frac{d r}{d t}=(\alpha+\beta+\delta+\gamma) i+(\beta+\delta) s-\alpha i s-\alpha i^{2}-(\beta+\delta)
\end{array}\right.
$$

Sendo N o número total de indivíduos, temos a seguinte equação diferencial para representação da variação temporal da população:

$$
\frac{d N}{d t}=\beta N-\mu N-\alpha i
$$

Supondo uma população de $N=11$ indivíduos que varia no decorrer de 20 semanas. Para obtenção dos dados foi utilizado um algoritmo computacional baseado na linguagem $\mathrm{C} / \mathrm{C}++$, e as informações obtidas foram arquivados para elaboração dos gráficos na plataforma Origin.

\section{Resultados e discussões}

Analisando o comportamento do sistema e considerando qualquer uma das duas hipóteses, observou-se dois pontos de equilíbrio. Sendo $\Theta$ o número de contato modificado do modelo (Oliveira, 2008), tem-se que o primeiro ponto de equilíbrio é o da não doença, $P_{1}(1,0,0)$, tendo como condição $\Theta>1$, provado pelo princípio de invariância de LaSalle. O segundo é o ponto de equilíbrio endêmico, $P_{2}(1,1,0)$, sendo $\Theta>1$, e com $0 \leq i \leq 1$, temos a condição de equilíbrio endêmico $\frac{\beta+\gamma}{\lambda-\alpha}<s<\frac{1}{\Theta}$.

Foram utilizados os valores $\lambda=0.233, \delta=0.098, \gamma=0.677, \mu=0.0001, \beta=0.003$ e $\alpha=$ 0 (Tsujiguchi, 2010) para a primeira hipótese. Pela simulação computacional do modelo SIRS com parâmetros da Influenza $\mathrm{A}(\mathrm{H} 1 \mathrm{~N} 1)$ obtivemos resultados que são apresentados na Figura 1 e Figura 2. Na segunda hipótese foram utilizados os mesmos valores, porém $\alpha=0.03$. Os resultados dessa segunda simulação são apresentados nas Figura 3 e Figura 4.

Na Figura 1, observa-se que a epidemia acaba antes da população ser extinta. O número de infectados cresce até atingir uma taxa máxima $c=3.92$, e logo depois decresce, tendendo à 


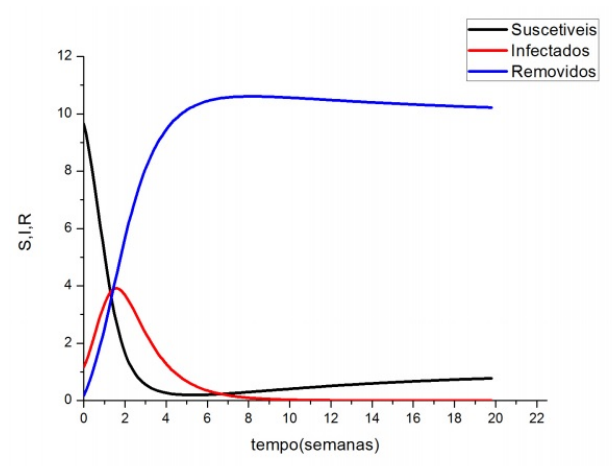

(a) Figura 1- Variação SIRS ao longo do tempo, $\operatorname{com} \alpha=0$.

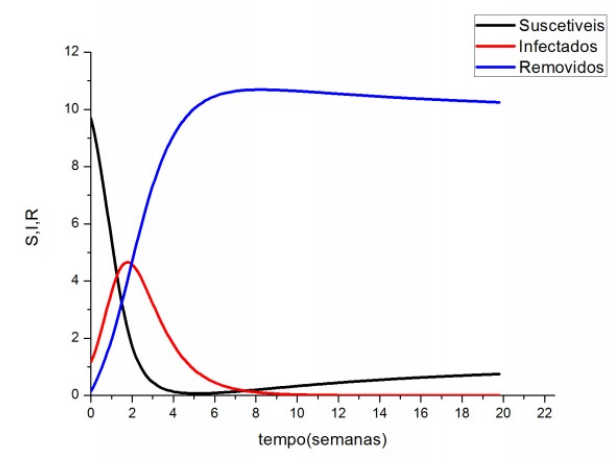

(c) Figura 3- Variação SIRS ao longo do tempo, com $\alpha \neq 0$.

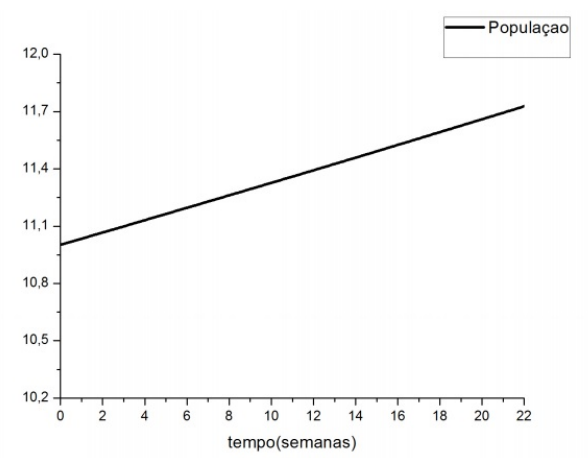

(b) Figura 2- População variando ao longo do tempo $\operatorname{com} \alpha=0$.

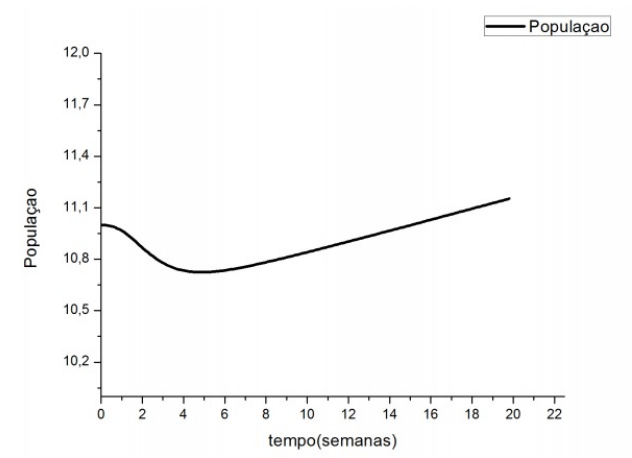

(d) Figura 4- População variando ao longo do tempo, $\operatorname{com} \alpha \neq 0$.

zero. A população de suscetíveis tem comportamento crescente até atingir uma taxa mínima de indivíduos suscetíveis $s=0.19$, sofrendo após isso um pequeno crescimento e apresentando um caráter constante. Por outro lado, observa-se um crescimento exponencial da taxa de indivíduos removidos sendo seu valor máximo $r=10.60$, ocorrendo após isso um pequeno decaimento e uma estabilização de forma assintótica. Os pontos $c, s$ e $r$ foram obtidos analisando os dados gerados pelo modelo.

A Figura 3 apresenta um comportamento semelhante ao da Figura 1, porém com resultados diferentes devido a inserção de uma taxa de mortalidade pela doença. Obtendo como taxa máxima de indivíduos infectados $c=3.08$, taxa mínima de indivíduos suscetíveis $s=0.07$ e taxa máxima de indivíduos removidos $r=10.69$.

Nas Figuras 2 e 4, observa-se que a taxa de variação total da população ao longo do tempo cresce atingindo um valor de $p=11.65$ na Figura 2 e $p=11.15$ na Figura 4, observa-se uma diferença devido a inserção da taxa de mortalidade pela doença. O comportamento da população descrito acima é tanto biologicamente aceitável como esperado de uma epidemia.

Observa-se, a partir das Figuras, que o parâmetro $\alpha$ está diretamente ligado ao comportamento da população dos infectados e no número total da população, pois afeta a dinâmica de natalidade e mortalidade da epidemia.

O modelo SIRS mostra-se, então, uma boa alternativa para o estudo epidemiológico por abranger diversos aspectos da epidemia e apresentar resultados biologicamente aceitáveis.

\section{Referências}

[1] Oliveira, I.M. (2008), Modelos Epidemiológicos SEIR, Tese de Doutorado, Departamento de Matemática Pura/Universidade do Porto, Tese de Mestrado, Portugal.

[2] Tsujiguchi, A.C. (2010), Um modelo de EDO aplicado à Influenza A(H1N1), UEMS, Dourados-MS. 